\title{
Changes in SDS Solubility of Glutenin Polymers during
}

\author{
Dough Mixing and Resting ${ }^{1,2}$
}

Thierry Aussenac ${ }^{3,4}$, Jean-Luc Carceller ${ }^{3}$, and Didier Kleiber ${ }^{3}$

${ }^{1}$ Research supported by a grant from Conseil Régional Midi-Pyrénées, Contract RECH 9507769.

${ }^{2}$ This work is based on a poster presented at the AACC Annual Meeting, october 31-3 november 1999, Seattle, USA.

${ }^{3}$ Department of Plant Physiology, E.S.A. Purpan, 75 voie du TOEC, 31076 Toulouse cedex 03 France.

${ }^{4}$ Corresponding author. Fax ; E-mail : aussenac@esa-purpan.fr 


\section{ABSTRACT}

An on-line coupling of HPSEC-MALLS and a RP-HPLC procedure were used to characterize and to reveal the polydispersity of the glutenin polymers of doughs during mixing and resting. Experiments involved doughs prepared from several samples of a common French wheat cultivar (Soissons) differing in total amount of SDS-unextractable glutenin polymers. During dough mixing the amounts, the size distribution of protein and the glutenin subunit composition within the SDS-unextractable polymers changed. However, the major changes in SDS-unextractable glutenin content and size distribution occurred before the peak MT was reached, while detectable changes in subunit composition occurred also after the peak MT. Even if sonication, which was used to solubilize the total wheat glutenin, can narrow down the glutenin size distribution, HPSEC-MALLS revealed a close relationship between the SDS solubility of the glutenin polymers and their size distribution confirming a depolymerization and repolymerization hypothesis. During the depolymerization of the SDSunextractable polymers, glutenin subunits were released in nonrandom order, which was indicative of the polymers' having a hierarchical structure. Some HMW-GS (specially HMWGS 1Dx5) were particularly resistant to the depolymerization mechanism. This suggested that the subunit plays a major role in forming the backbone of the SDS-unextractable polymers consistent with its potential to form branched structure. These studies suggest that the SDSunextractable polymers in flours have a well-ordered structure that can be modified by dough mixing and resting. 


\section{INTRODUCTION}

Proteins are recognized as the most important component governing bread-making quality (Schofield and Booth 1983, Shewry and Miflin 1985, Wrigley and Bietz 1988). The large variation in dough strength and bread-making performances of wheat flours can indeed, to a large extent, be ascribed to variations in the level and quality of gluten proteins (Finney 1943, Finney and Barmore 1948). However, among the different flour protein group, the glutenin fraction (a complex group of polypeptides joined together by interpolypeptide disulfide bonds) is the most important fraction related to bread-making quality (MacRitchie et al 1990). For this reason, in the last decades, the polymeric glutenin fraction has been investigated intensively (Weegels et al 1996). Although the precise structure of these polymers is still a matter of speculation, it has been shown that interchain disulfide (S-S) bridges link high molecular weight glutenin subunits (HMW-GS) and low molecular weight glutenin subunits (LMW-GS) into polymers with varying molecular weight (MacRitchie 1992). The total glutenin polymer quantity is known to be correlated with various technological parameters (Huebner and Wall 1976, Field et al 1983, Dachkevitch and Autran 1989, Singh et al 1990a,b). Moreover, a certain amount of these polymers remain unextractable in various extracting systems (e.g. acetic acid solution or SDS phosphate buffer). Those unextractable polymeric proteins appear also to be correlated with baking performance (Kurowska and Bushuk 1988, Gupta et al 1993, Jia et al 1996a). Besides, Gupta et al (1992) showed that the unextractable polymer quantity is more directly linked with certain technological parameters (especially those correlated with mixing) than the total glutenin quantity. The proportion of unextractable polymer fraction among the glutenin polymers appears likewise to be an important ratio for technological response (Gupta et al 1993, Jia et al 1996b). The bread-making process phase where gluten functionality can be deemed to be critical is that of dough development. Several researchers have found that the 


\section{Cereal Chemistry}

T. Aussenac et al. 4

1 quantity of unextractable glutenin polymer decreases during dough mixing and increases

2 again during dough resting, although the nature of the polymer present before mixing is different from that present afterwards (Mecham et al 1962 ; Tsen 1967 ; Tanaka and Bushuk 1973a,b,c ; Graveland et al 1980 ; Danno and Hoseney 1982a ; Weegels et al 1994,1995 ; Veraverbeke et al 1999 ; Skerritt et al 1999). Different mechanisms have been proposed to explain these observations. It has been suggested that, during mixing, the size of protein aggregates decreases (Mecham et al 1965, Tsen 1967) by physical separation of the aggregates (Tsen 1967) and/or by breaking of non-covalent (Tsen 1967) or covalent bonds (Graveland et al 1980, Danno and Hoseney 1982b, Tanaka and Bushuk 1973c, MacRitchie 1975). Hamer and Lichtendonk (1987), who have reported a decrease in extractability of glutenin during resting, have assumed that re-polymerisation of glutenin polymer takes place.

The current study, which used several samples of a common French wheat cultivar (Soissons) differing in total amount of unextractable glutenin polymers, aimed to identify possible relationships between the stage of dough mixing and resting and changes in the content and molecular size distribution of glutenin polymers. An on-line coupling of HPSECMALLS (Multiangle Laser Light Scattering) was used to reveal the polydispersity of the glutenin polymers during the process. Changes in the glutenin subunit composition of these unextractable polymers were also investigated by RP-HPLC during dough mixing and resting. 


\section{Flour samples and mixing properties}

Several grain samples of the common wheat cultivar Soissons (harvest 1996) were milled into flours on a Miag Multomat mill and used for mixing experiments. The compositions of flours are given in Table I.

Mixing properties were determined with a recording Mixograph using $10 \mathrm{~g}$ of flour. The same amount of water was added as needed to give a Farinograph consistency of $500 \pm$ 15 BU (ICC standard 115, see Table I) according to Weegels et al (1995). To follow the behavior of the unextractable glutenin polymers, doughs were mixed for various times $(1,2$, $3,4,5,6,8$ or $10 \mathrm{~min}$ ) at a temperature of $25^{\circ} \mathrm{C} \pm 1.0^{\circ} \mathrm{C}$. Directly after mixing, or after 45 , 90 or135 min resting at $30{ }^{\circ} \mathrm{C}$ in capped bottles to prevent drying, doughs were frozen in liquid nitrogen. All doughs that were frozen in liquid nitrogen were freeze-dried. The freezedried doughs were finely ground in a Janke A10 grinder fitted with a $200 \mu \mathrm{m}$ screen.

\section{SDS unextractable glutenin polymer quantification}

Freeze-dried doughs $\left(240 \mathrm{mg}\right.$ ) were stirred for $2 \mathrm{hr}$ at $60^{\circ} \mathrm{C}$ in $30 \mathrm{~mL}$ of $0.1 \mathrm{M}$ sodium phosphate buffer ( $\mathrm{pH}$ 6.90) containing $2 \%$ (w/v) SDS. These extractions were followed by centrifugation during $30 \mathrm{~min}$ at $12,500 \times \mathrm{g}$ at $20^{\circ} \mathrm{C}$ in a Jouan centrifuge (model MR 1822). Clears supernatants (soluble proteins) were eliminated and pellets (unextractable glutenin polymers) were freeze-dried. The Dumas method (AOAC 7.024) was used to determine the nitrogen concentration of freeze-dried pellets. Three or four replicates were done and combined for analysis. Protein concentration was determined on a Leco apparatus (model FP 428) by multiplying $\mathrm{N}$ values by 5.7 . 
1 Extraction and purification of glutenin polymers

Freeze-dried doughs $(0.1 \mathrm{~g})$ were stirred for $1 \mathrm{~h}$ at room temperature $\left(25^{\circ} \mathrm{C}\right)$ with $2 \mathrm{~mL}$ of $0.3 \mathrm{M}$ Sodium Iodide (NaI) in $7.5 \%(\mathrm{v} / \mathrm{v})$ of propan-1-ol according to $\mathrm{Fu}$ and Kovacs (1999). Extraction was followed by centrifugation at $15900 \times \mathrm{g}$ for $15 \mathrm{~min}$ at $15^{\circ} \mathrm{C}$. The supernatant (mainly monomeric proteins) was eliminated. The glutenins remain unextractable in the sodium iodide. The pellet was washed twice with the solvent of extraction and was then used for HPSEC-MALLS procedure.

\section{Molecular size distribution of glutenin polymers by HPSEC-MALLS}

The residue $(0.1 \mathrm{~g})$ obtained during the purification procedure was extracted with 1 $\mathrm{mL}$ of a sodium phosphate buffer $0.1 \mathrm{M}(\mathrm{pH} 6.90)$ containing $2 \%$ (w/v) SDS and was sonicated for $15 \mathrm{~s}$ at power setting of $50 \%$ (output $10 \mathrm{~W}, 23 \mathrm{kHz}$ ) using a stepped microtip probe (3 mm diameter) (Branson Sonifier, model B-12) and centrifuged for $30 \mathrm{~min}$ at 12500 $g$.

The HPSEC system comprised a SpectraSYSTEM LC (Thermo Separation Products S.A., Les Ulis, France) consisting of on-line degasser (model SCM 400), isocratic pump (model P 4000), variable auto-injector (model AS 3000) and variable wavelength detector (model UV 2000). Refractive index of solutes was carried out with a differential refractive index detector (Erma ERC-7512). Light scattering was measured on a Wyatt multi angle laser light scattering detector (laser wavelength $=632.8 \mathrm{~nm})($ model Dawn-F, Wyatt Technology Corporation, Santa Barbara, CA). Analog signals from the 15 photodiode channels were sent to a PC with an on-board 16 channel A/D converter. The $16^{\text {th }}$ channel was used for the signal of the mass-sensitive detector. Photodiode coefficients were normalised using bovine serum albumin monomer as a reference. The size-exclusion system comprised two columns in series : a PL aquagel-OH $60(300 \times 7.5$ mm I.D., $8 \mu \mathrm{m})$ (Polymer Laboratories Ltd., 
1 Shropshire, UK) and a PL aquagel-OH $40(300 \times 7.5 \mathrm{~mm}$ I.D., $8 \mu \mathrm{m})$ (Polymer Laboratories

Ltd., Shropshire, UK). The eluant was sodium phosphate buffer $0.1 \mathrm{M}(\mathrm{pH}$ 6.90) with $0.1 \%$ (w/v) SDS, carefully degassed and filtered before use through $0.1 \mu \mathrm{m}$ membranes (Gelman Sciences, France). The flow rate was $0.7 \mathrm{~mL} / \mathrm{min}$. During the fractionation , the columns were thermostated at $25^{\circ} \mathrm{C}$. Samples $(100 \mu \mathrm{L})$ were injected into the HPSEC system.

Multi-angle laser scattering means measuring the intensity of the scattered light emitted by the sample molecules at different scattering angles $(\theta)$. With a modern MALLS photometer, it is possible to continuously monitor the scattering by means of several detectors mounted at different angles. For each elution slice, a weight average molar mass $\left(M_{W}\right)$ can be calculated using the following equations :

$$
\begin{array}{cc}
P(\theta)=1-a_{1}[2 k \sin (\theta / 2)]^{2}+a_{2}[2 k \sin (\theta / 2)]^{4}-\ldots & \text { Eq. } 1 \\
\frac{K c}{R_{\theta}}=\frac{1}{M_{W} P(\theta)}+2 A_{2} c+\ldots & \text { Eq. } 2 \\
k=\frac{4 \pi^{2} n_{0}^{2}}{N_{A} \lambda_{0}^{4}}\left(\frac{d n}{d c}\right)^{2} & \text { Eq. } 3
\end{array}
$$

where $K$ is a light scattering constant, containing the wavelength $\lambda_{0}$ of the incident light, the refractive index $n_{0}$ of the pure eluent, and the refractive index increment $\mathrm{d} n / \mathrm{d} c, c$ is the concentration, $A_{2}$ is the second virial coefficient, $R_{\theta}$ is the excess Rayleigh ratio, and $P(\theta)$ is a general form of a scattering function. For very low concentrations, the second and higher order terms in equation 1 can be neglected, and $R_{\theta}$ becomes directly proportional to $M_{\mathrm{W}} P(\theta)$. Plotting $R_{\theta} / K c$ against $\sin ^{2}(\theta / 2)$ gives $M_{W}$ from the intercept with the ordinate ; from the angular dependence of the intensity of the scattered light, which is included in $a_{1}$ and higher order terms of the equation 2, a $z$-average root mean square radius $\left\langle R_{\mathrm{G}}{ }^{2}\right\rangle_{\mathrm{z}}{ }^{0.5}$ can be derived. 
1 This latter quality is defined in terms of the distribution of the volume elements of the

2 molecule with the respect to the square of the distance from its center of gravity :

$$
\left\langle R_{G}^{2}\right\rangle_{z}^{0.5}=\frac{1}{V} \int r^{2} d V
$$

5 The calculation of the values $\left\langle R_{\mathrm{G}}^{2}\right\rangle_{\mathrm{z}}^{0.5}$ is independant of $\mathrm{d} n / \mathrm{d} c, M_{W}$ and even $c$ and therefore 6 is insensible to errors. Number-average $\left(\langle M\rangle_{\mathrm{n}}\right)$, weight average $\left(\langle M\rangle_{\mathrm{W}}\right), z$-average $\left(\langle M\rangle_{\mathrm{z}}\right)$ 7 molecular weights, $\left\langle R_{\mathrm{G}}{ }^{2}\right\rangle_{\mathrm{z}}{ }^{0.5}$ and polydispersity index $\left(P=\langle M\rangle_{\mathrm{W}} /\langle M\rangle_{\mathrm{n}}\right)$ were established with ASTRA ${ }^{\circledR}$ software using a Zimm extrapolation $\left(K c / R_{\theta}\right)$. Normalisation of the photodiodes (i. e., the process by which the various photodiodes' signals are related to the $90^{\circ}$ detector signal) was obtained using a relatively narrow (i. e., $\langle M\rangle_{\underline{w}} /\langle M\rangle_{n} \approx 1$ ) low molecular weight

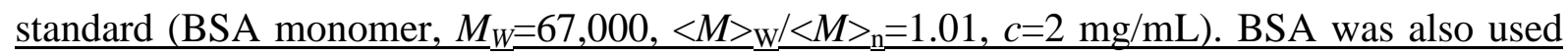
to determine the interconnection volume between detectors to $0.172 \mathrm{~mL}$. A value of 0.190 $\mathrm{mL} / \mathrm{g}$ was employed as refractive index increment $(\mathrm{d} n / \mathrm{d} c)$ for glutenins.

\section{Quantification of glutenin subunits by RP-HPLC}

Freeze-dried unextractable glutenin polymers $(0.5 \mathrm{~g})$ were extracted successively with station (Spectra-Physics analytical software, San Jose, CA) in conjunction with a Zorbax 300 


\section{Cereal Chemistry}

T. Aussenac et al. 9

1 Bellefont, PA]) preceded by a guard column of the same packing material (Zorbax $300 \mathrm{CB}$ -

$2 \mathrm{C} 8,2-\mathrm{cm}, 4.6 \mathrm{~mm}$ i.d.) were used for analysis of glutenin subunits. Column temperature was 3 maintained at $50^{\circ} \mathrm{C}$; the column effluent was monitored at $210 \mathrm{~nm}$. Glutenin subunits were

4 resolved as described previously ( $\mathrm{Jia}$ et al 1996a). Injection volume were $30 \mu \mathrm{L}$ in 5 conjunction with a multiple $5 \mu \mathrm{L}$ injection technique (Marchylo and Kruger 1988). 


\section{Changes in SDS-unextractable polymer content during mixing and resting}

It is well known that extractability of flour proteins increases when mixing flour with water into a dough (see review by Weegels et al. 1996 ). This can be observed in figure 1a as a decrease in the amount of the SDS-unextractable glutenins from dough during mixing as determined by the Dumas protein analysis of the SDS-unextractable residue. Furthermore, the amount of SDS-unextractable glutenin polymer decreases exponentially during the mixing and this decrease is independent of the flours used (i.e. from the SDS-unextractable glutenin polymer content of the flour). All the curves can indeed be plotted well $\left(r^{2}=0.9688\right)$ by using a unique exponential regression (Fig. 1b). A $70 \%$ reduction of the level of SDS-unextractable glutenins was observed during mixing to peak consistency (first $4 \mathrm{~min}$ ). Further mixing resulted in no significant decrease in the level of SDS-unextractable proteins. However, the decrease in SDS-unextractable glutenin content began well before mixograph peak time was reached. These results suggest, as previously presented by Skerritt et al. (1999), that the reduction in quantity of these polymers may not be linked directly to dough breakdown (the period after peak mixing resistance is reached and during which time dough resistance shows a continuous decrease with further mixing). Similar results were obtained by Pritchard and Brock (1994). Notwithstanding, as has been previously shown (Graveland et al 1980 ; Danno and Hoseney 1982a ; Weegels et al 1994 ; Veraverbeke et al 1999 ; Skerritt et al 1999), the extractability of glutenin polymers decreased during resting (Fig. 1a). Although there is some restoration of the level of the SDS-unextractable glutenins, a similar level to that which is to be found in flour is not attained during resting. Even if no definite conclusions have been reached, the depolymerization and re-polymerization mechanism have been proposed to 
1 explain these observations (Mecham et al 1965, Tsen 1967, Tanaka and Bushuk 1973c,

2 MacRitchie 1975, Hamer and Lichtendonk 1987).

As previously shown by Weegels et al. (1994), the amount of SDS-unextractable glutenin polymers during the process (mixing and resting) can be very well predicted on the basis of SDS-unextractable glutenin polymer content of flour and mixing and resting time only. The data presented in figures $2 \mathrm{a}, \mathrm{b}$ confirms this for Soissons doughs. The amount of SDS-unextractable glutenins in dough directly after mixing (DIP) can indeed be described by an exponential function of the amount of SDS-unextractable glutenins in flour (FIP) and the times of mixing time (MT) (Fig. 2a). With this equation, $87 \%$ of the variation in polymer content can be explained. Using the results obtained from resting, a relationship between the amount of SDS-unextractable glutenins in dough and the amount of SDS-unextractable glutenins in flour can be found. The amount of SDS-unextractable glutenins in dough after resting (DIPR) can be well described (i.e., 66\% of variation) by the amount of SDSunextractable glutenins in flour (FIP) and resting time (RT) (Fig. 2b). According to Weegels et al. (1994, 1996) who used different genotypes, it is clear that all the modifications of the glutenin polymers occurring during the process are mainly governed by quantity and far less by quality differences. This probably indicates that glutenin subunits and notably HMW-GS are related indirectly to breadmaking quality via the quantity and molecular weight distribution of glutenins polymers.

\section{Changes in molecular weight distribution of the glutenin polymers}

Even if no definite conclusions have been reached to explain the modifications in glutenins extractability during both dough mixing and resting, different mechanisms have been proposed. All these hypotheses were mainly based on a decrease of the glutenin polymer size by physical separation of the aggregates (Tsen 1967) and/or by breaking of non-covalent 
1 (Tsen 1967) or covalent bonds (Danno and Hoseney 1982, Tanaka and Bushuk 1973,

2 MacRitchie 1975, Graveland et al. 1980). In the current study, a one-line coupling of HPSEC-

3 MALLS was used to reveal changes in molecular size distribution of the glutenin polymers

4 during mixing.

The results of HPSEC-MALLS experiments on the polymeric glutenins of Soissons T7 flour are shown in figure 3a,b. Figure 3a shows a MALLS/RI chromatogram of total polymer separated by HPSEC illustrating the remarkable power of light scattering detection for studying the high molecular weight fraction. In the same figure the molar masses of the polymeric glutenins determined by means of light scattering are represented as a function of the elution volume. The molar mass distributions (differential and cumulative representation) represented in figure $3 \mathrm{~b}$ were calculated by including the concentration of each respective species. From these distributions it was possible to determine the number-average $\left(\langle M\rangle_{\mathrm{n}}\right)$, weight-average $\left(\langle M\rangle_{\mathrm{w}}\right)$ and $\mathrm{z}$-average $\left(\langle M\rangle_{\mathrm{z}}\right)$ molar mass, and also the polydispersity index $\left(P=\langle M\rangle_{\mathrm{w}} /\langle M\rangle_{\mathrm{n}}\right)$. All these measurements of polymeric glutenins show that the multi-angle laser light-scattering photometer is suitable for monitoring the eluate from the HPSEC on the condition that any exclusion limit happens on the gel matrix, as is the case with the SEC stationary phases (PL aquagel-OH 40 and 60) used here. Consequently, the angular-dependent scattered light readings yield the absolutely radius of gyration and molar mass and, by including the respective concentrations, they also yield the distributions for these two variables.

The increase in SDS solubility of the unextractable glutenin polymers was highly correlated with the reduction of the number-average $\left(\langle M\rangle_{n}\right)$ and weight-average molar mass $\left(\langle M\rangle_{\mathrm{w}}\right.$ ) of these proteins during mixing (Fig. 4). In fact, both the variables (UG and $\langle M\rangle$ ) can be plotted well by using exponential regression as a function of mixing time (MT). From these results it appears that the SDS extractability of the glutenin polymers during mixing to 


\section{Cereal Chemistry}

T. Aussenac et al.

1 the dough peak consistency (first $4 \mathrm{~min}$ ) can be the result of the size reduction of these

2 glutenin polymers confirming the depolymerisation hypothesis. In flour, the molar masses and

3 the radii of gyration of the total polymers are roughly in agreement with a size distribution of glutenin calculated by Ewart (1987) based on standard theory of high polymers assuming linear molecules. The most probable weight fraction had a molecular weight of $\approx 1.0 \mathrm{E}+6$ $\mathrm{g} / \mathrm{mol}$, and molecular weights extended to $>5.0 \mathrm{E}+6 \mathrm{~g} / \mathrm{mol}$. Recent measurements obtained by flow FFF have also pointed to molecular weights in millions (Wahlund et al. 1996, Stevenson and Preston 1996, Stevenson et al. 1999). These authors utilized the observed hydrodynamic diameters $(d)$ to estimate the upper and lower limits for the molecular mass of glutenins. The lower limit was defined as $d=0.0542 M^{0.498}$ for flexible random coil polymer, and the upper $\underline{\text { limit was defined as } d=0.159 M^{0.333} \text { corresponding to a spherical shape. Values for the upper }}$ limit were in the range of 440,000 to $11 \mathrm{E}+6 \mathrm{~g} / \mathrm{mol}$. Like in the present study, because no solvent has been found for solubilizing the total wheat glutenin, the solutions have been 1998, Carceller 2000). However, as we have already shown (Carceller 2000)., even if sonication can narrow down the glutenin size distribution, the protocol applied in the present study is largely insufficient to reach a molecular distribution limit. Consequently, it does enable the following of modifications in molecular distribution directly linked to dough mixing.

During mixing, the relative amount of the highest molecular weight fraction decreased, while the lowest molecular weight fraction, possibly corresponding to the oligomers, increased (Fig. 5a,b). 59.1\% of flour total polymers have a molar mass which is greater than $1.0 \mathrm{E}+6 \mathrm{~g} / \mathrm{mol}$ while they represent only $47 \%$ in the dough after 4 min of mixing. At the same time, oligomers characterized by a molar mass lower than $4.0 \mathrm{E}+5 \mathrm{~g} / \mathrm{mol}$ represent $14.8 \%$ and $24.2 \%$ in flour and dough after 4 min of mixing respectively. These 
1 results are in total agreement with the observations made by Borneo and Khan (1999) and

2 Skerritt et al. (1999) using a multilayer SDS-PAGE procedure. After 4 min of mixing (peak

3 MT), the results revealed a depolymerisation limit (i.e. a $\langle M\rangle_{\mathrm{n}}$ and $\langle M\rangle_{\mathrm{w}}$ limit) (Fig. 4).

Indeed, the number-average $\left(\langle M\rangle_{\mathrm{n}}\right)$ and weight-average molar mass $\left(\langle M\rangle_{\mathrm{w}}\right)$ of the glutenin polymers were not significantly different between 4 and $10 \mathrm{~min}$ of mixing. These observations suggested that a critical size distribution had been reached. The changes in size distribution that have been observed for glutenin polymers correspond closely to those predicted by the Bueche theory (1960). In fact, the theory of Bueche predicts that for a polymer with a wide molecular weight distribution the chain degradation by shear only occurs at practical shear rates if entanglements are present. Breakage of bonds arises because molecular chains cannot disentangle sufficiently rapidly in response to the stress shear. The highest tension occurs at the center of the chain and, as a result, chains break preferentially at their centers. That way, all chains with a molecular mass higher than a critical size will be broken down and none of the low molecular mass material will be broken.

Even if the study of this depolymerisation phenomenon deserves greater attention, the overall observations made which are in total agreement with previous works (Gupta et al. 1992, Gupta et al. 1993, MacRitchie and Lafiandra 1997, Bangur et al 1997), nevertheless, confirm the existence of a close link between glutenin molecular distribution and rheological dough properties. Therefore, in agreement with the first remarks made by Bangur et al (1997), it seems that only polymeric proteins above a certain molecular size contribute to dough properties such as dough strength. This behavior is also in line with the theory of Bersted and Anderson (1990) (Eq. 5). In this theory, based on a modified version of the Flory equation (1945), Bersted and Anderson suggest that for polydispersed polymers only those molecules that formed effective entanglements contribute to tensile strength $(\sigma)$ : 
where $\sigma_{0}=$ the limiting tensile strength at high molecular weight; $M_{\mathrm{T}}=$ the threshold

molecular weight for effective entanglements; $\phi=$ the fraction with molecular weight $>M_{\mathrm{T}}$; $M_{\mathrm{n}}{ }^{*}=$ the number-average molecular weight of this fraction (i.e. the fraction with molecular weight $>M_{\mathrm{T}}$ ).

\section{Changes in glutenin subunit composition during mixing and resting}

In the present study, it was found that the decrease in the amount of SDSunextractable glutenin polymers during dough mixing was accompanied by changes in the composition of these polymers (i.e. relative proportions of different glutenin subunits) (Fig. 6a,b). A significant increase in the proportion of HMW-GS in SDS-unextractable polymers was observed during mixing to peak dough consistency (first 4 min) (Fig. 6a). Further mixing resulted in a decrease of the HMW-GS level in the SDS-unextractable polymers. The proportion of HMW-GS in the unextractable glutenins that was reached at the end of mixing was not significantly different to the proportion observed in flour. Finally, this HMW-GS proportion remained closely constant during the dough resting. These observations seem to demonstrate that the polymers more resistant to solubilization by dough mixing were enriched in HMW-GS. Therefore, the HMW-GS proportion in SDS-unextractable polymers decreases after peak MT, suggesting the reduction of these subunits may be linked to dough breakdown. Then, during dough resting the glutenins re-polymerization did not induce any modification of the relative subunit composition.

Apart from the changed HMW-GS/LMW-GS ratio, the different HMW-GS did not disappear at the same rate from the SDS-unextractable glutenins resulting in changed related proportions of the various HMW-GS. These observations were well illustrated in figure 7, 


\section{Cereal Chemistry}

T. Aussenac et al.

1 which shows reversed-phase HPLC patterns of HMW-GS from dough at different mixing and

2 resting time. Skerritt et al. (1999) observed similar effects. However, their observations that

3 the $\mathrm{x}$-type HMW-GS solubilise more readily than y-type HMW-GS could not be confirmed in the present study. Indeed, with Soissons flours it was found that the relative proportion of HMW-GS 1Dy10 decreased while the proportion of HMW-GS 1Dx5 increased during mixing (Fig. 6b). At the same time, the proportion of HMW-GS 1Bx7, 1By8 and 1A2* remained relatively constant. These observations were in total agreement with other works (Veraverbeke et al. 1999). However, in our study, all the significant modifications of relative proportions observed for HMW-GS 1Dx5 and 1Dy10 occurred after the peak MT (dough breakdown) and not during mixing to peak dough consistency (first $4 \mathrm{~min}$ ).

Interpretation of the changes in HMW-GS composition of the SDS-unextractable glutenin polymers during dough mixing and resting is difficult since the mechanisms responsible for the changes in the amount of SDS-unextractable glutenin are poorly understood. However, from our results it appears that glutenin subunits (HMW-GS, LMWGS, x- and y-type HMW-GS) were released from the polymers in a non-random order, which was indicative of the polymers having a hierarchical structural organization. The data obtained is in keeping with a proposed model for polymers, in which a backbone of HMWGS exists to which LMW-GS are bound (Graveland et al. 1985). According to Skerritt et al. (1999), we cannot exclude the fact that differences in depolymerization rates between LMWGS and HMW-GS can be explained by the presence of polymers with differing composition or branching within SDS-unextractable polymers; or within SDS-unextractable polymers by the presence of different classes of glutenin subunits inducing an unevenly distributed branching. Furthermore, our results, which demonstrate the major role of HMW-GS and/or xtype HMW-GS to the formation of the SDS-unextractable polymers, are very consistent with the observations made by Linsay and Skerritt (1998). These authors, using stepwise reduction 


\section{Cereal Chemistry}

T. Aussenac et al.

1 to examine the structure of SDS-unextractable glutenins, suggest that these polymers have a

2 well-ordered structure in which some HMW-GS (particularly HMW-GS 1Dx5) play a major

3 role in forming the backbone to them. Kasarda (1999) has indeed proposed a hierarchical

4 arrangement of the glutenin subunits that represents their relative potentials for the glutenin

5 polymer formation. This hierarchical arrangement of glutenin subunits in which $\mathrm{x}$-type

6 HMW-GS are defined as the most important constituent, is based on a combination of two

7 factors relating to a) the potential of a subunit to form branched polymers, as opposed to

8 linear polymers, and $b$ ) the length of the repeating sequence domain. 
During dough mixing the amounts, the size distributions of protein and the glutenin subunit composition within the SDS-unextractable polymers changed. However, the major changes in SDS-unextractable glutenin content and size distribution occurred before the peak MT was reached, while detectable changes in subunit composition occurred also after the peak MT. HPSEC-MALLS, which was very sensible to changes in polymer molecular weight distribution, revealing a close relationship between the SDS solubility of the glutenin polymers and their size distribution. Consequently, modifications of the SDS solubility of the glutenin polymers during the process (mixing and resting) can be attributed to a large extent to modifications of size distribution confirming the depolymerization and repolymerization hypothesis.

Depolymerization of SDS-unextractable polymers during mixing to peak consistency (first 4 min in the present study) which was characterized by a significant variation of the HMW-GS/LMW-GS ratio was indicative of the polymers having a hierarchical structural organization. In fact, HMW-GS were apparently more resistant to the depolymerization than LMW-GS, consistent with them forming a backbone to the SDS-unextractable glutenin polymers. Furthermore, from our results it appears that the LMW-GS released may be unevenly linked with HMW-GS. During mixing to peak consistency, the HMW-GS 1Dx5 was indeed not released at the same rate as the other HMW-GS from the SDS-unextractable polymers consistent with them forming mainly HMW-GS linkage. After peak MT the decrease of the HMW-GS/LMW-GS ratio induced with further mixing may be related directly to dough breakdown (overmixed doughs). During this step, the unextractable glutenin polymers become enriched in some HMW-GS (specially HMW-GS 1Dx5) suggesting that this subunit plays a major role in forming the backbone of the SDS-unextractable polymers consistent with its potential to form branched structures (Kasarda 1999). 


\section{Cereal Chemistry}

T. Aussenac et al.

The data obtained is in keeping with a proposed model for polymers, in which a

2 backbone of HMW subunits exists to which LMW subunits are bound (Graveland et al. 1985)

3 but in which clusters of glutenin polymers are linked by rheologically effective disulfide

4 bonds (Gao et al. 1992). This may be relevant to the observation reported by several workers

5 that a relatively small fraction of the total number of disulfide bonds presents only some

6 disulfide contribute to rheological properties.

7 
Bangur, R., Batey, I. L., McKenzie, E., and MacRitchie, F. 1997. Dependence of extensograph parameters on wheat protein composition measured by SE-HPLC. J. Cereal Sci. 25:237-245.

Bersted, B. H., and Anderson, T. G. 1990. Influence of molecular weight and molecular weight distribution on the tensile properties of amorphous polymers. J. Appl. Polym. Sci. 39:499-514.

Borneo, R., and Khan, K. 1999. Glutenin protein changes during breadmaking of four spring wheats : fractionation by multistacking SDS gel electrophoresis and quantification with high-resolution densitometry. Cereal Chem. 76:718-726.

Bueche, F. 1960. Mechanical degradation of high polymers. J. Appl. Polym. Sci. 4:91-100.

Carceller, J. -L. 2000. Formation, accumulation, et caractérisation des polymères gluténiques du grain de blé tendre (Triticum aestivum L.). Thèse de Doctorat, INP Toulouse. 215 pp.

Dachkevitch, T., and Autran, J. C. 1989. Prediction of baking quality of bread wheats in breeding programs by size-exclusion high-performance liquid chromatography. Cereal Chem. 66:448-456.

Danno, G., and Hoseney, R. C. 1982a. Changes in flour proteins during dough mixing. Cereal Chem. 59:249-253.

Danno, G., and Hoseney, R. C. 1982b. Effects of dough mixing and rheologically active compounds in relative viscosity of wheat proteins. Cereal Chem. 59:196-198.

Field, J. M., Shewry, P. R., and Miflin, B. J. 1983. Solubilisation and characterisation of wheat gluten proteins : correlations between the amount of aggregated proteins and baking quality. J. Sci. Food Agric. 34:370-377. 
1 Finney, K. F. 1943. Fractionating and reconstituting techniques as tools in wheat flour research. Cereal Chem. 20 :381-396.

Finney, K. F., and Barmore, M. D. 1948. Loaf volume and protein content of hard winter and spring wheats. Cereal Chem. $25: 291-312$.

Flory, P. J. 1945. Tensile strength in relation to molecular weight of high polymers. J. Am. Chem. Soc. 67:2048-2050.

Fu, B. X., and Sapirstein, H. D. 1996. Procedure for isolating monomeric proteins and polymeric glutenin of wheat flour. Cereal Chem. 73:143-152.

Fu, B. X., and Kovacs, M. I. P. 1999. Rapid single-step procedure for isolating total glutenin proteins of wheat flours. J. Cereal Sci. 29:113-116.

Gao, L., Ng, P. K. W., and Bushuk, W. 1992. Structure of glutenin based on farinograph and electrophoresis results. Cereal Chem. 69:452-455.

Graveland, A., Bosveld, P., Lichtendonk, W. J., and Moonen, J. H. E. 1980. Superoxide involvement in the reduction of disulfide bonds of wheat gel proteins. Biochem. Biophy. Res. Comm. 93:1189-1195.

Graveland, A, Bosveld, P., Lichtendonk, W. J., Marseille, G. J., Moonen, J. H. E., and Scheepstra, A. 1985. A model for molecular structure of glutenins from wheat flour. J. Cereal Sci. 3:1-15.

Gupta, R. B., Batey, I. L., and MacRitchie, F. 1992. Relationship between protein composition and functional properties of wheat flours. Cereal Chem. 69:125-131.

Gupta, R. B., Khan, K., and MacRitchie, F. 1993. Biochemical basis of flour properties in bread wheats. I. Effects of variation in the quality and size distribution of polymeric protein. J. Cereal Sci. 18:23-41.

Hamer, R. J., and Lichtendonk, W. J. 1987. Structure-function studies on gluten proteins. Reassembly of glutenin proteins after mixing. Pages 227-237. In : Proceedings of the $3^{\text {rd }}$ 


\section{Cereal Chemistry}

T. Aussenac et al.

International Workshop on Gluten Proteins. Eds. Lasztity, R., and Bekes, F., World Scientific Publishers, Singapore.

Huebner, F. R., and Wall, J. S. 1976. Fractionation and quantitative differences of glutenin from wheat varieties varying in baking quality. Cereal Chem. 53:258-269.

Jia, Y. Q., Masbou, V., Aussenac, T., Fabre, J. -L., and Debaeke, P. 1996a. Effects of nitrogen fertilization and maturation conditions on protein aggregates and on breadmaking quality of Soissons, a common wheat cultivar. Cereal Chem. 73:123-130.

Jia, Y. Q., Fabre, J. -L., and Aussenac, T. 1996b. Effects of growing location on response of protein polymerization to increased nitrogen fertilization for the common wheat cultivar Soissons : relationship with some aspects of the bread-making quality. Cereal Chem. 73:526-532.

Kasarda, D. D. 1999. Glutenin polymers : the in vitro to in vivo transition. Cereal Food World 44, 566-571.

Kurowska, E., and Bushuk, W. 1988. Solubility of flour and gluten protein in a solvent of acetic acid, urea, and cetyltrimethylammonium bromide, and its relationship to dough strength. Cereal Chem. 65:156-158.

Linsay, M. P., and Skerritt, J. H. 1998. Examination of the structure of the glutenin macropolymer in wheat flour and doughs by stepwise reduction. J. Agric. Food Chem. 46:3447-3457.

MacRitchie, F. 1975. Mechanical degradation of gluten proteins during high-speed mixing of doughs. J. Polymer Sci.49:85-90.

MacRitchie, F., DuCross, D. L., and Wrigley, C. W. 1990. Flour polypeptides related to wheat quality. Pages 79-115 in: Advances in Cereal Science and Technology, Vol X. Y. Pomeranz, ed. Am. Assoc. Cereal Chem. :St. Paul. MN. 
1 MacRitchie, F. 1992. Physicochemical properties of wheat proteins in relation to functionality. Adv. Food Nutrition Res. 36 :1-87.

MacRitchie, F., and Lafiandra, D. 1997. Structure-fonction relationships of wheat proteins. Pages 293-323 in: Food Proteins and Their Applications. S. Damodaran and A. Paraf, eds. Marcel Dekker: New York.

Marchylo, B. A, and Kruger, J. E. 1988. The effect of injection volume on the quantitative analysis of wheat storage proteins by reversed-phase high-performance liquid chromatography. Cereal Chem.65:192-198.

Marchylo, B. A., Kruger, J. E., and Hatcher, D. W. 1989. Quantitative reversed-phase highperformance liquid chromatographic analysis of wheat storage proteins as a potential quality prediction tool. J. Cereal Sci. 9:113-130.

Mecham, D. K., Sokol, H. A., and Pence, J. W. 1962. Extractable protein and hydration characteristics of flours and doughs in dilute acid. Cereal Chem. 39:81-93.

Mecham, D. K., Cole, E. G., and Pence, J. W. 1965. Dough-mixing properties of crude and purified glutens. Cereal Chem. 42:409-420.

Pritchard, P. E., and Brock, C. J. 1994. The glutenin fraction of wheat protein : The importance of genetic background on its quantity and quality. J. Sci. Food Agric. 65:401-406.

Skerritt, J. H., Hac, L., and Bekes, F. 1999. Depolymerisation of the glutenin macropolymer during dough mixing. I. Changes in levels, molecular weight distribution, and overall composition. Cereal Chem. 76:395-401.

Singh, N. K., Donovan, R., and MacRitchie, F. 1990a. Use of sonication and size-exclusion high-performance liquid chromatography in the study of wheat flour proteins. I. Relative quantity of glutenin as a measure of breadmaking quality. Cereal Chem. 67 : 161-170. 
Singh, N. K., Donovan, R., and MacRitchie, F. 1990b. Use of sonication and size-exclusion high-performance liquid chromatography in the study of wheat flour proteins. II. Dissolution of total proteins in the absence of reducing agents. Cereal Chem. $67: 150$ 161.

Southan, M. D., Batey, I. L., and MacRitchie, F. 1998. Fractionation of wheat endosperm proteins by flow field-flow fractionation. Pages $9-11$ in : Proc. $48^{\text {th }}$ Australian Cereal Chemistry Conf. RACI : Melbourne.

Stevenson, S. G., and Preston, K. R. 1996. Flow field-flow fractionation of wheat proteins. J. Cereal Sci. $23: 121-131$.

Stevenson, S. G., Ueno, T., and Preston, K. R. 1999. Automated frit inlet/frit outlet flow fieldflow fractionation for protein characterisation with emphasis on polymeric wheat proteins. Anal. Chem. $71: 8-14$.

Tanaka, K., and Bushuk, W. 1973a. Changes in flour proteins during dough-mixing. I. Solubility results. Cereal Chem. $50: 590-596$.

Tanaka, K., and Bushuk, W. 1973b. Changes in flour proteins during dough-mixing. II. Gel filtration and electrophoresis results. Cereal Chem. 50 :597-605.

Tanaka, K., and Bushuk, W. 1973c. Changes in flour proteins during dough-mixing. III. Analytical results and mechanisms. Cereal Chem. 50 :606-612.

Tsen, C. C. 1967. Changes in flour proteins during dough mixing. Cereal Chem. 44 :308-317.

Veraverbeke, W. S., Courtin, C. M., Verbruggen, I. M., and Delcour, J. A. 1999. Factors governing levels and composition of the sodium dodecyl sulphate-unextractable glutenin polymers during straight dough breadmaking. J. Cereal Sci. 29 :129-138.

Wahlund, K. -G., Gustavsson, M., MacRitchie, F., Nylander, T., and Wannerberger, L. 1996. Size characterisation of wheat proteins, particularly glutenin, by asymmetrical flow field-flow fractionation. J. Cereal Sci. $23: 113-119$. 


\section{Cereal Chemistry}

T. Aussenac et al.

1 Weegels, P. L., Hamer, R. J., and Schofield, J. D. 1994. Depolymerisation and repolymerisation of individual glutenin subunits in situ in dough - Implications for the structure of gluten. Pages 57-66 in: Gluten proteins, 1993. Assoc. Cereal Res. : Detmold, Germany.

Weegels, P. L., Orsel, R., Van de Pijpekamp, A. M., Lichtendonk, W. J., Hamer, R. J., and Schofield, J. D., 1995. Functional properties of low $M_{\mathrm{r}}$ wheat proteins. II. Effects on dough properties. J. Cereal Sci. 21 :117-126.

Weegels, P. L., Hamer, R. J., and Schofield, J. D. 1996. Critical review : Functional properties of wheat glutenin. J. Cereal Sci. 23 :1-18. 
Fig. 1 (A) Effects of mixing $[\mathrm{M}]$ and resting $[\mathrm{R}]$ on the SDS-unextractable glutenin polymer 9

content and (B) level of SDS-unextractable glutenins (UG) in dough as a function of mixing time (MT) of several Soissons flours differing in total amount of SDS-unextractable glutenins. ( $\square, \mathbf{0})$ SoissonsT1, $(\square, \bigcirc)$ SoissonsT2, $(\square, \mathbf{x})$ SoissonsT3, $(\square, \bullet$ ) SoissonsT4, $(\square, \square)$ SoissonsT5, $(\square,+)$ SoissonsT6, $(\square, \Delta)$ SoissonsT7 for (A) and (B) respectively.

Fig. 2 (A) Predicted dough SDS-unextractable polymer (DIP) content by mixing time (MT) and flour SDS-unextractable polymer content (FIP) and, (B) predicted amount of SDSunextractable polymers in dough after resting (DIPR) by resting time (RT) and flour SDSunextractable polymer content (FIP). (O) SoissonsT1, (O) SoissonsT2, ( $\mathbf{X}$ ) SoissonsT3, ( • ) SoissonsT4, ( $\square)$ SoissonsT5, (+) SoissonsT6, ( $\Delta$ ) SoissonsT7.

Fig. 3 (A) Steric-exclusion chromatography and (B) hydrodynamic parameters of total glutenin polymers of SoissonsT7. (A) RI detection (fine line), light scattering at $90^{\circ}$ (bold line) and molar mass as a function of elution time ( $\bullet$ ). (B) Cumulative (fine line) and differential (bold line) distribution of molar mass.

Fig. 4 Level of SDS-unextractable glutenins (UG) and changes in number-average $\left(\langle M\rangle_{\mathrm{n}}\right)$ molecular weight of these polymers as a function of mixing time (MT). 
1 Fig. 5 (A) Differential and (B) cumulative distribution of molar mass of total glutenin

2 polymers during mixing. (๑) Flour, (○) 2 min mixing, (• ) 3 min mixing, (*) 4 min mixing,

$3 \quad(\Delta) 5$ min mixing and, ( $\square) 8$ min mixing.

4

5 Fig. 6 (A) Changes in HMW-GS/LMW-GS ratio (bold line) and, (B) relative proportions of the different HMW-GS in total HMW-GS in the SDS-unextractable glutenins during mixing $[\mathrm{M}]$ and resting $[\mathrm{R}] .(\bigcirc) 1 \mathrm{Dx} 5,(\bigcirc) 1 \mathrm{Dy} 10,(\Delta) 1 \mathrm{Bx} 7,(\bullet) 1 \mathrm{Ax} 2 *$ and, $(\diamond) 1 \mathrm{By} 8$.

9 Fig. 7 Reversed-phase high-performance liquid chromatography patterns of HMW-GS in SDS-unextractable polymers during mixing $[\mathrm{M}]$ and resting $[\mathrm{R}]$. 
TABLE I

Composition and properties of flours

4

\begin{tabular}{|c|c|c|c|c|}
\hline Samples & $\begin{array}{c}\text { Moisture } \\
\text { Content } \\
(\%, w / w)\end{array}$ & $\begin{array}{l}\text { Protein } \\
\text { content }^{\mathrm{a}} \\
(\%, \mathrm{w} / \mathrm{w})\end{array}$ & $\begin{array}{c}\text { SDS- } \\
\text { unextractable } \\
\text { glutenin content }{ }^{\mathrm{a}} \\
(\%, \mathrm{w} / \mathrm{w})\end{array}$ & $\begin{array}{c}\text { Brabender water } \\
\text { absorption }^{\mathrm{b}} \\
(\%)\end{array}$ \\
\hline Soissons T1 & 13.55 & 7.9 & 1.9 & 53.4 \\
\hline Soissons T2 & 13.84 & 8.6 & 2.2 & 53.4 \\
\hline Soissons T3 & 13.57 & 10.6 & 2.6 & 54.8 \\
\hline Soissons T4 & 13.97 & 10.1 & 2.3 & 54.5 \\
\hline Soissons T5 & 13.42 & 11.5 & 2.7 & 55.5 \\
\hline Soissons T6 & 13.26 & 11.3 & 2.8 & 55.6 \\
\hline Soissons T7 & 13.08 & 12.9 & 2.9 & 55.5 \\
\hline
\end{tabular}

${ }^{\mathrm{a}} \mathrm{N} \times 5.7$; results expressed on a dry-matter basis.

${ }^{\mathrm{b}}$ On flour basis according to ICC Standard 115. 


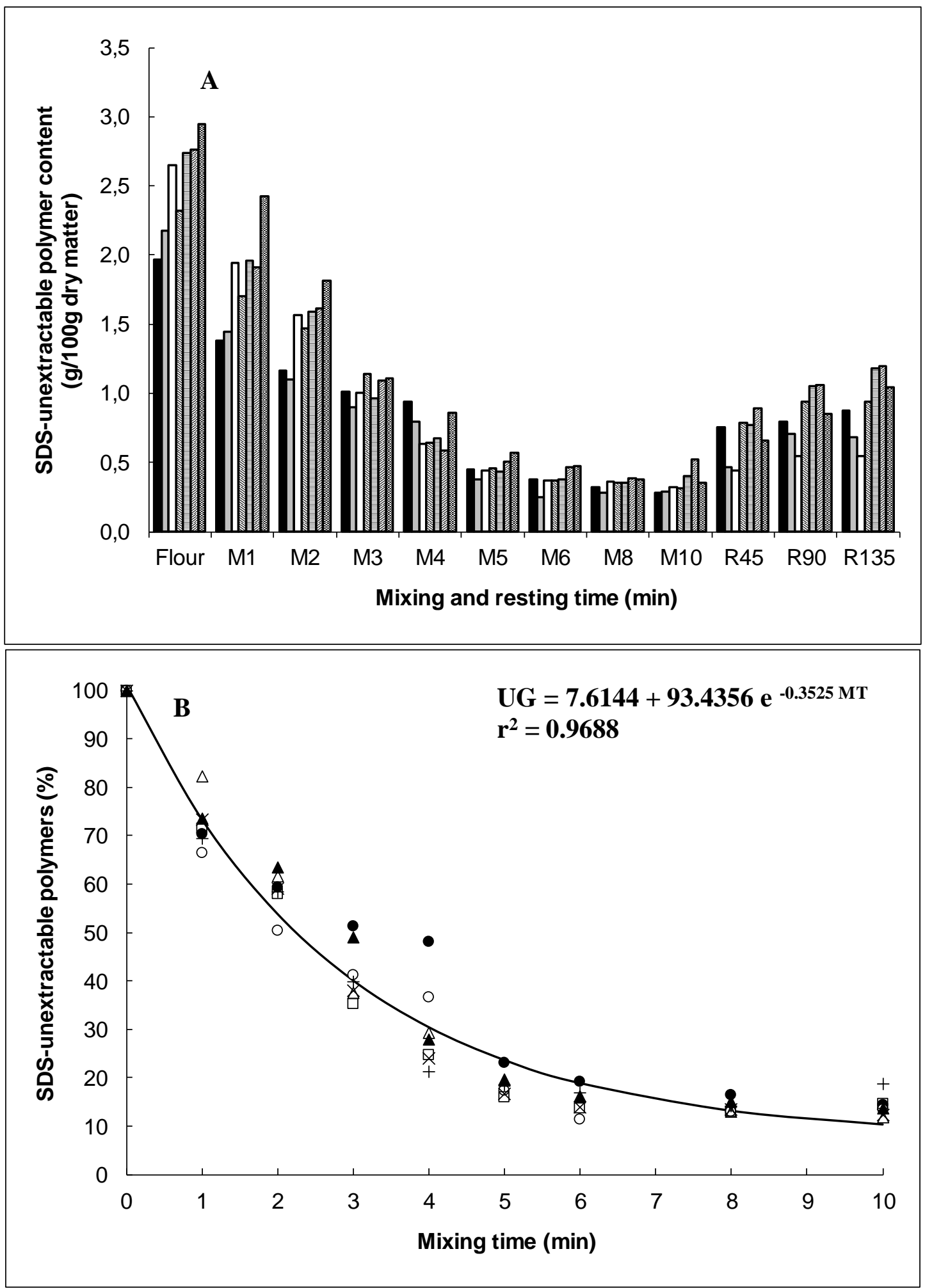

Fig. 1 

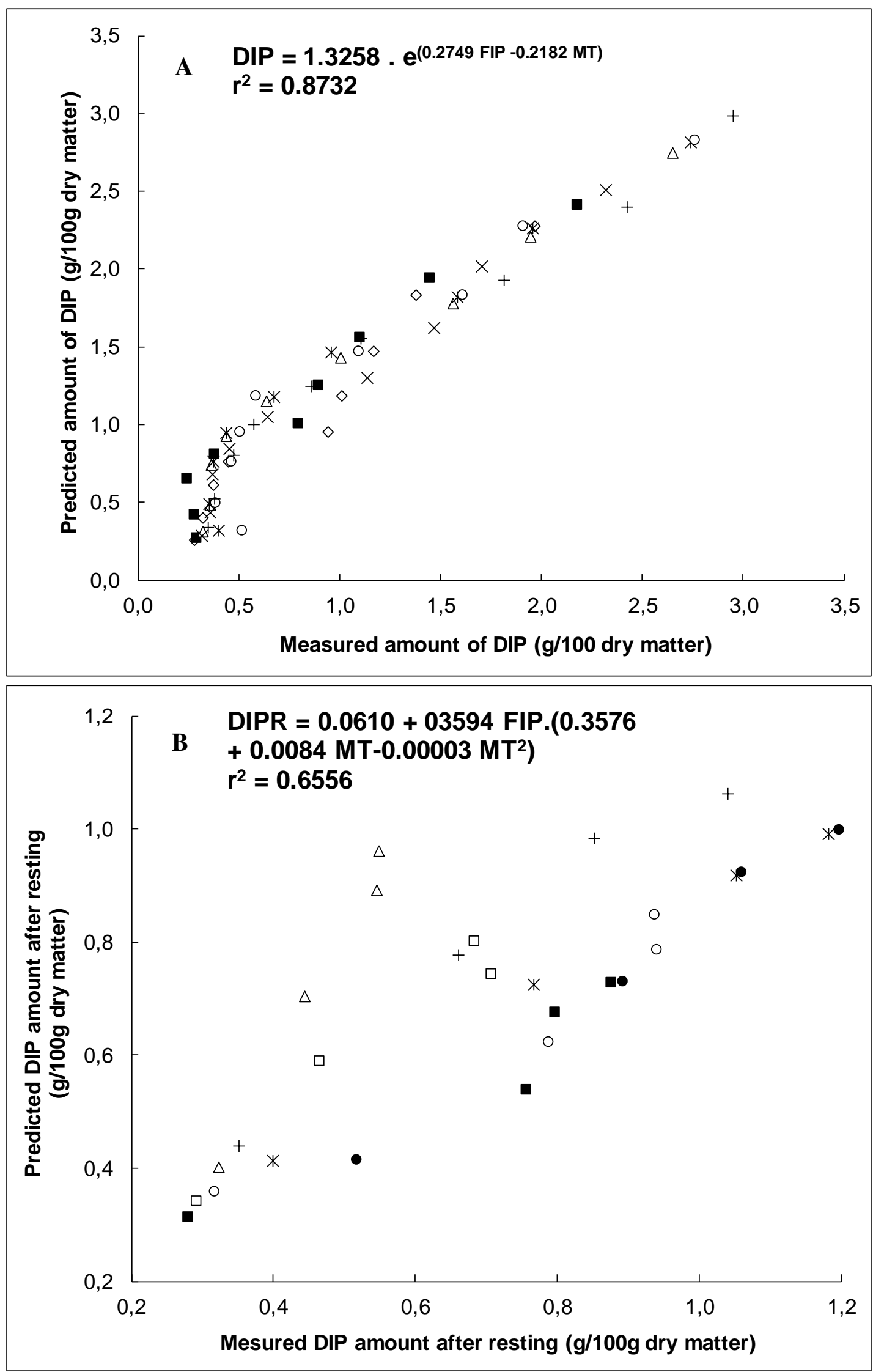

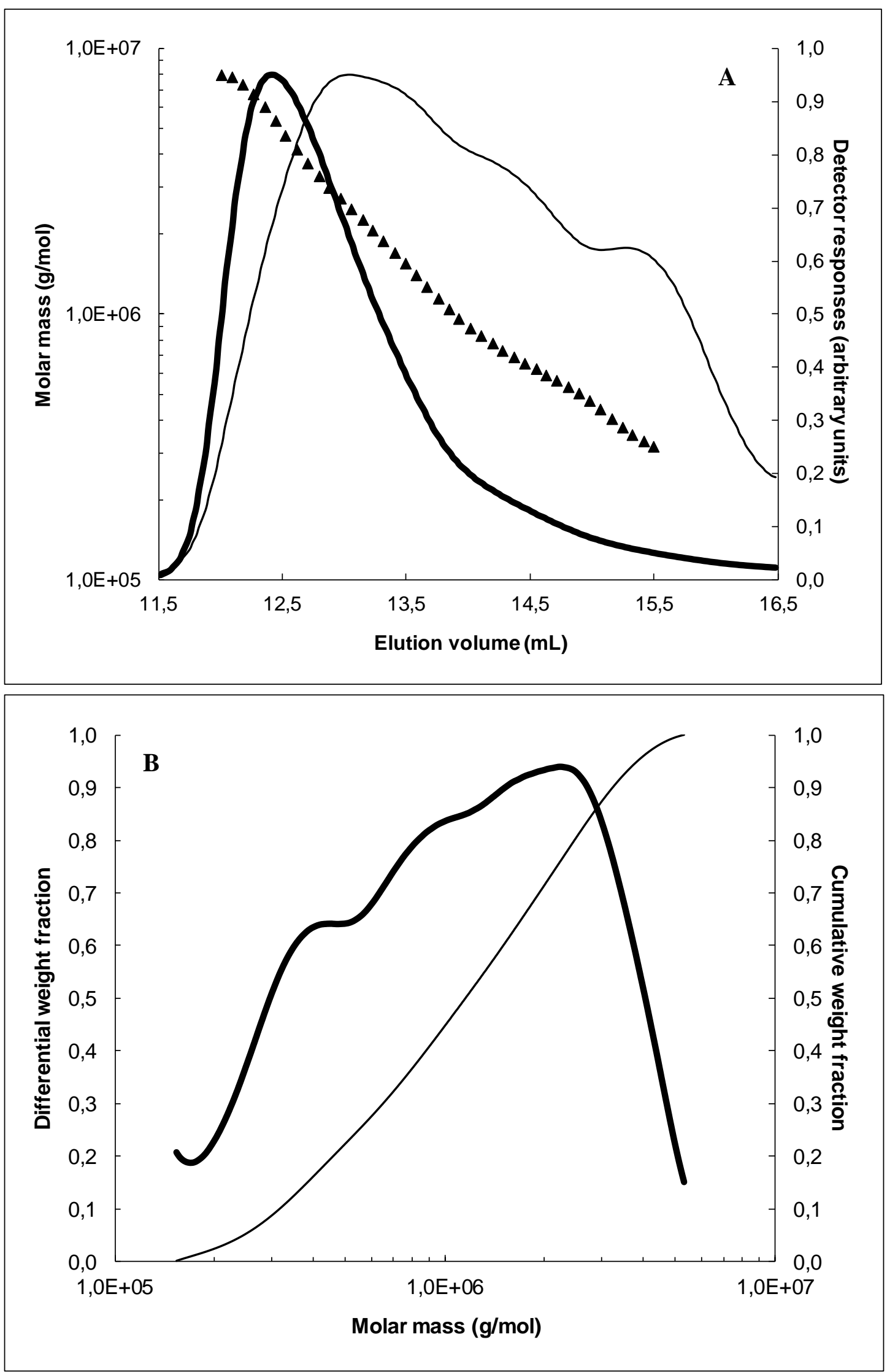

Fig. 3 


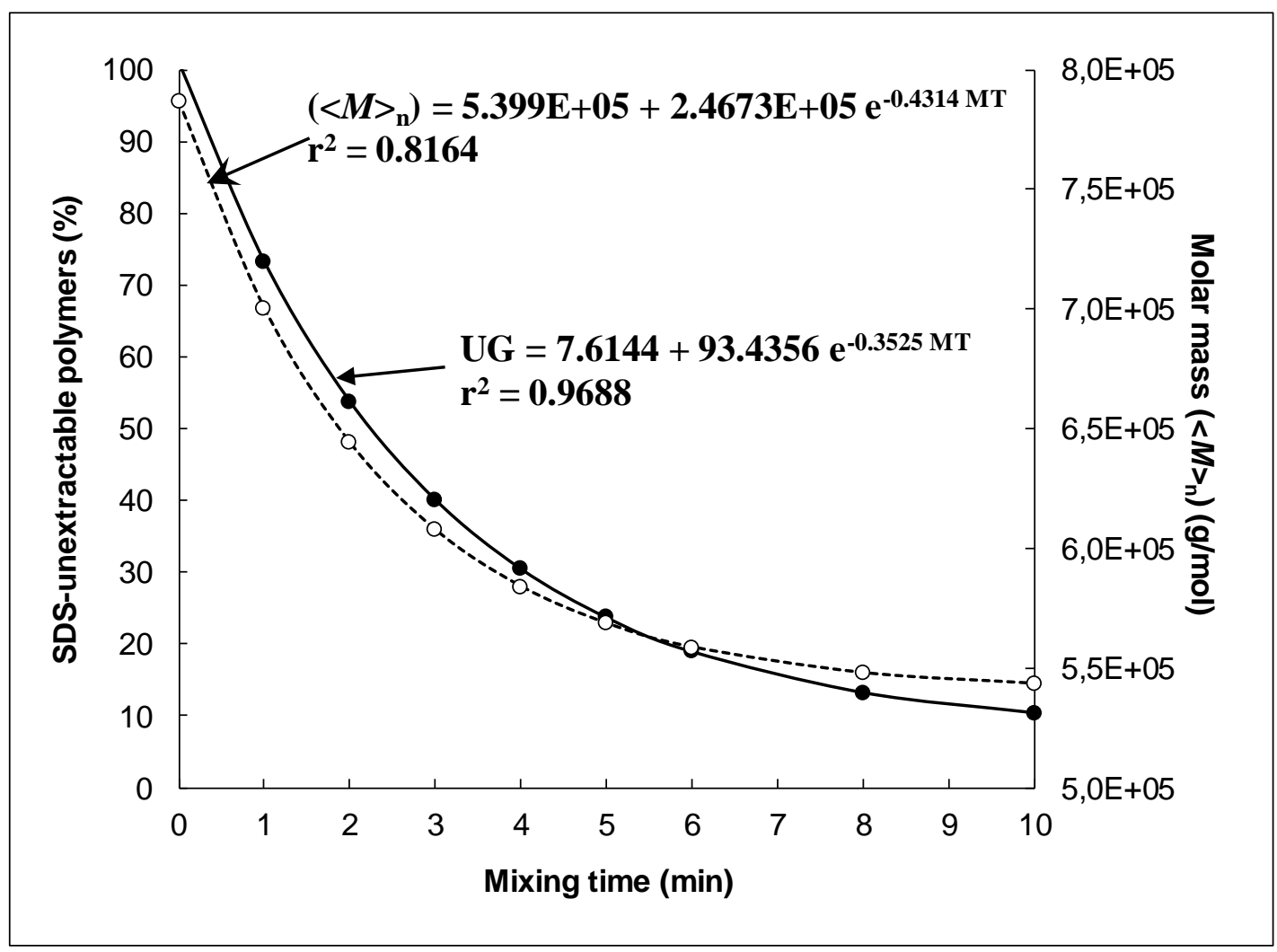

Fig. 4 

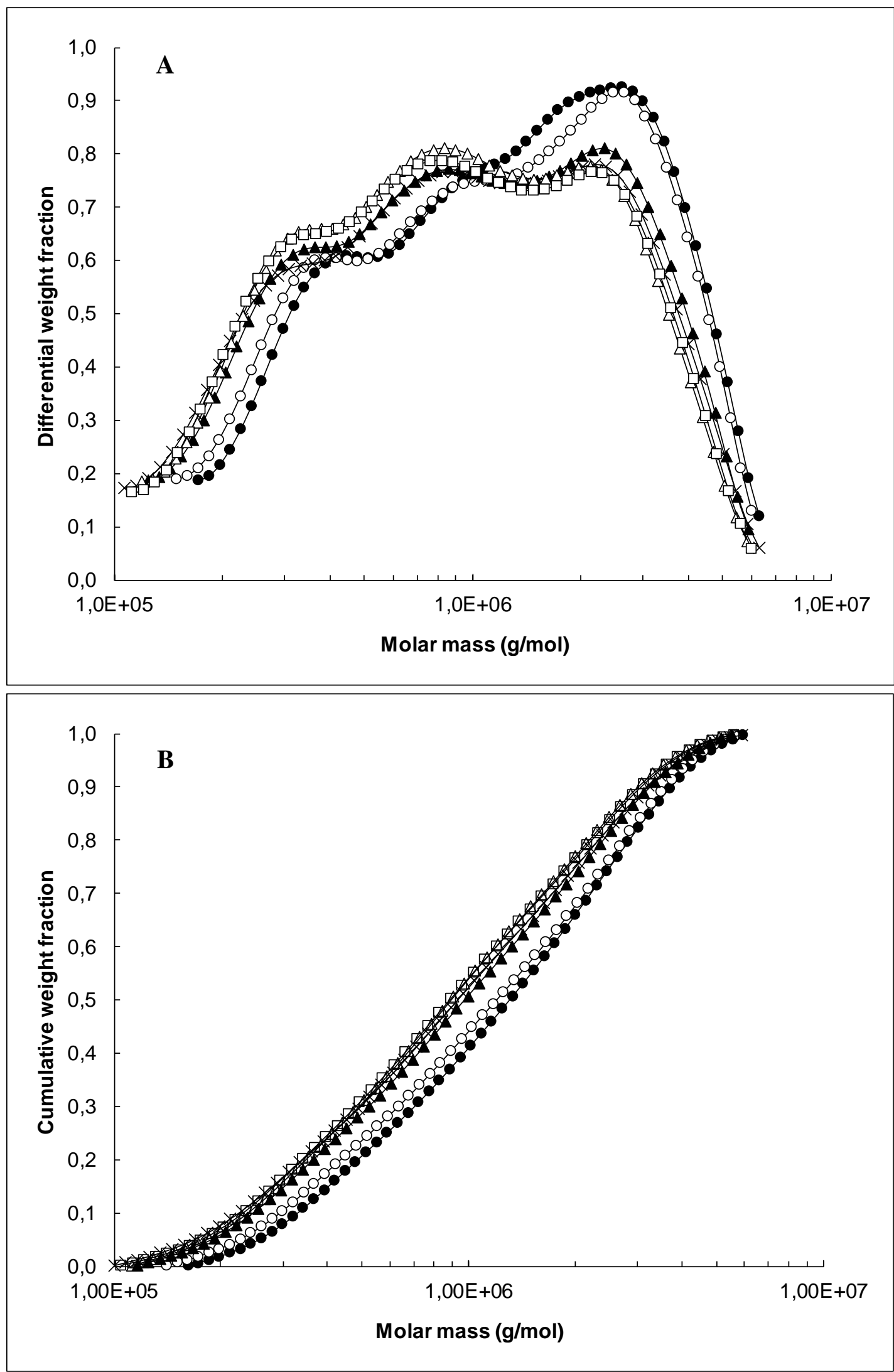

Fig. 5 

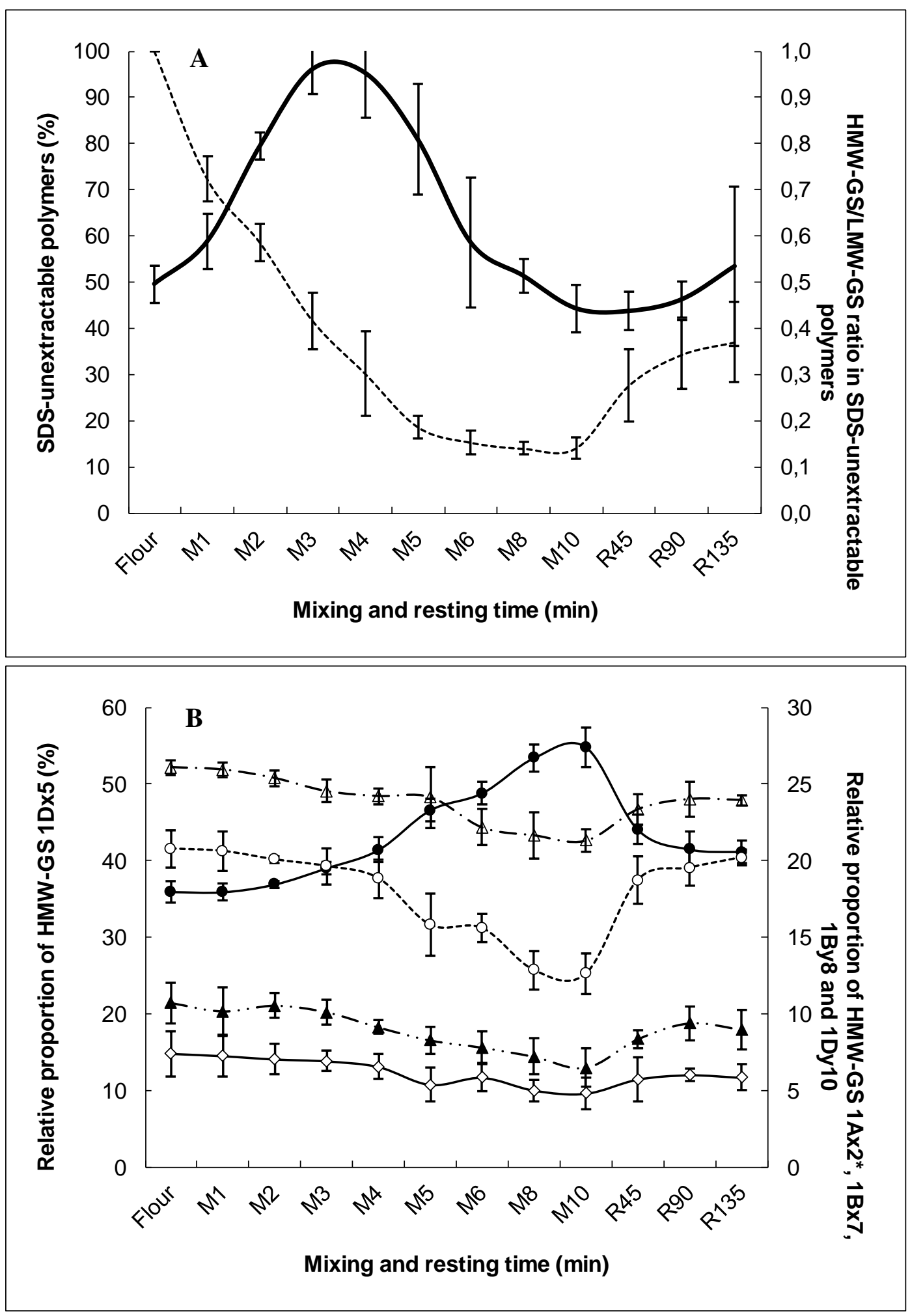

Fig. 6 


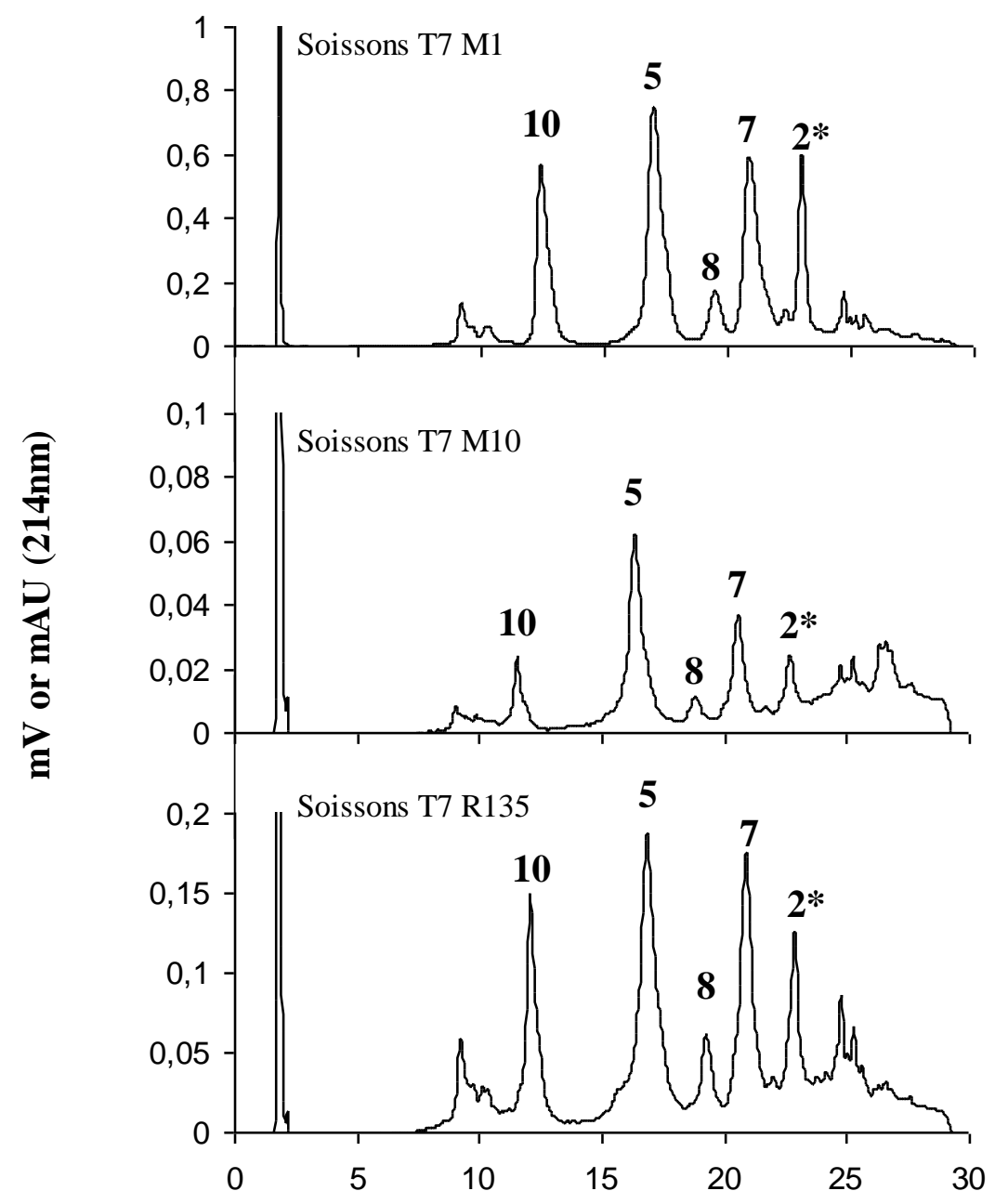

Elution time (mn)

Fig. 7 